\title{
Efeitos da Lidocaína por Via Venosa sobre a Farmacodinâmica do Rocurônio *
}

\section{Effects of Intravenous Lidocaine on the Pharmacodynamics of Rocuronium}

Leandro Sotto Maior Cardoso, TSA ${ }^{1}$; César Romão Martins, TSA ${ }^{2}$; Maria Angela Tardelli, TSA ${ }^{3}$

\section{RESUMO}

Cardoso LSM, Martins CR, Tardelli MA - Efeitos da Lidocaína por Via Venosa sobre a Farmacodinâmica do Rocurônio

JUSTIFICATIVA E OBJETIVOS: O rocurônio é um bloqueador neuromuscular (BNM) não-despolarizante de ação intermediária que apresenta início de ação mais rápido, indicado para situações onde há necessidade de intubação traqueal rápida. A lidocaína é freqüentemente utilizada por via venosa para diminuir as respostas hemodinâmicas associadas à intubação traqueal. A associação de um BNM a um anestésico local resulta em potencialização dos efeitos bloqueadores neuromusculares. O objetivo deste estudo é avaliar a interação entre a lidocaína e a farmacodinâmica do rocurônio avaliada por aceleromiografia.

MÉTODO: Pacientes estado físico ASA I e II, com idade entre 18 e 65 anos, aleatoriamente distribuídos em dois grupos (GC: controle e GL: lidocaína), receberam rocurônio como bloqueador neuromuscular. O GL recebeu lidocaína $(1,5$ $\left.\mathrm{mg} . \mathrm{kg}^{-1}\right) 3$ minutos antes do rocurônio. A função neuromuscular foi avaliada pela resposta do músculo adutor do polegar à seqüência de quatro estímulos (SQE). Após a injeção do BNM foram anotados os tempos para a primeira resposta $\left(T_{1}\right)$ até a SQE atingir $10 \%$ e $0 \%$ do valor controle, e recuperar $25 \%, 75 \%$ e $95 \%$ da altura de contração (Dur $25 \%$, Dur ${ }_{75 \%}$, Dur ${ }_{95 \%}$ ). Anotou-se também o tempo de recuperação de $\left.T_{4} / T_{1}=0,8\right)$ e os intervalos Dur ${ }_{75 \%}$-Dur $25 \%\left(I R_{25-75}\right)$ e $T_{4} / T_{1}=0,8-$ Dur $_{25 \%}$.

RESULTADOS: Este estudo não demonstrou diferença estatística significativa entre os grupos quando comparados $T_{1}=10 \%, T_{1}=0, I R_{25-75}, T_{4} / T_{1}=0,8-D_{1} r_{25}$. Os tempos para Dur $_{25 \%}$, Dur $75 \%$, Dur $95 \%$ no GL foram estatisticamente superiores aos do GC.

CONCLUSÕES: A associação de lidocaína ao rocurônio prolongou a fase inicial de recuperação do bloqueio sem interferir com o início de ação ou com a fase de recuperação final.

\footnotetext{
* Recebido da (Received from) Universidade Federal de São Paulo Escola Paulista de Medicina (UNIFESP - EPM), São Paulo, SP

1. Anestesiologista, Universidade Federal de São Paulo - Escola Paulista de Medicina

2. Anestesiologista, Preceptor dos Residentes do CET da UNIFESP-EPM

3. Professora Adjunta da Disciplina de Anestesiologia, Dor e Terapia Intensiva da UNIFESP - EPM
}

Apresentado (Submitted) em 20 de setembro de 2004

Aceito (Accepted) para publicação em 10 de março de 2005

Endereço para correspondência (Correspondence to)

Dra. Maria Angela Tardelli

Disciplina de Anestesiologia, Dor e Terapia Intensiva

Rua Napoleão de Barros, $715,4^{\circ} \mathrm{A}$

04024-002 São Paulo, SP

E-mail: tardelli.dcir@epm.br

(C) Sociedade Brasileira de Anestesiologia, 2005
Unitermos: ANESTÉSICOS, Local: lidocaína; BLOQUEADORES NEUROMUSCULARES, Não-Despolarizante: rocurônio

\section{SUMMARY}

Cardoso LSM, Martins CR, Tardelli MA - Effects of Intravenous Lidocaine on the Pharmacodynamics of Rocuronium

BACKGROUND AND OBJECTIVES: Rocuronium is an intermediate nondepolarizing neuromuscular blocker (NMB) with faster onset and indicated in situations requiring rapid tracheal intubation. Intravenous lidocaine is often used to decrease hemodynamic responses to tracheal intubation. The association of NMB to local anesthetics results in potentiation of NMB effects. The purpose of this study was to evaluate the influence of lidocaine on rocuronium's pharmacodynamics determined by acceleromyography.

METHODS: Forty-six ASA I-II patients, aged 18 to 65 years, were randomly distributed in two groups (CG: control and LG: lidocaine). Rocuronium was given to all patients for neuromuscular block. $L G$ received lidocaine $\left(1.5 \mathrm{mg}^{-\mathrm{kg}^{-1}}\right) 3$ minutes before rocuronium. Neuromuscular function was evaluated by adductor pollicis muscle response to TOF. After NMB injection, times for first TOF response $\left(T_{1}\right)$ to reach 10 and $0 \%$ of baseline value and recover $25 \%, 75 \%$ and $95 \%$ of contraction height (Dur $25 \%$, Dur $75 \%$, Dur $95 \%$ ) were recorded. Recovery time of $T_{4} / T_{1}=0.8$ and intervals Dur ${ }_{75 \%}-\operatorname{Dur}_{25 \%}\left(I R_{25-75}\right)$ and $T_{4} / T_{1}=0.8-$ Dur $_{25 \%}$ were also recorded.

RESULTS: This study has not shown statistically significant differences between groups when $T_{1}=10 \%, T_{1}=0, R I_{25-75}, T_{4} / T_{1}=$ 0.8 - Dur $25 \%$ were compared. Times for Dur $25 \%$, Dur $75 \%$, Dur ${ }_{95 \%}$ in $L G$ were significantly higher as compared to CG.

CONCLUSIONS: Lidocaine associated to rocuronium has prolonged early blockade recovery stage without interfering with onset or late recovery stage.

Key Words: ANESTHETICS, Local: lidocaine; NEUROMUSCULAR BLOCKERS: Nondepolarizing: rocuronium

\section{INTRODUÇÃO}

$\mathrm{O}$ brometo de rocurônio é um bloqueador neuromuscular (BNM) não-despolarizante aminoesteróide de ação intermediária ${ }^{1-5}$. Entre os BNM não-despolarizantes de uso clínico, o rocurônio é o que apresenta início de ação mais curto $^{6}$, sendo indicado quando se deseja intubação traqueal rápida sem os inconvenientes da succinilcolina ${ }^{7}$.

A lidocaína é um anestésico local, freqüentemente utilizado por via venosa durante a indução anestésica, com a finalidade de diminuir as respostas hemodinâmicas associadas à intubação traqueal ${ }^{8}$.

Há evidências de que os anestésicos locais diminuem a transmissão neuromuscular e exercem efeitos no neurônio 
motor e na fibra muscular. Estudos in vitro mostraram que a associação desse fármaco com um BNM resulta em potencialização dos efeitos bloqueadores neuromusculares ${ }^{9,10}$. Na prática clínica, esse efeito sinérgico entre BNM e anestésico local poderia ser interessante se resultasse em instalação mais precoce do bloqueio neuromuscular, o que possibilitaria uma intubação traqueal mais rápida aliada à proteção dos efeitos cardiovasculares inerentes à técnica.

O objetivo deste estudo foi avaliar se a administração por via venosa de lidocaína, antes da administração de rocurônio para a intubação traqueal, modifica a farmacodinâmica deste bloqueador neuromuscular.

\section{MÉTODO}

Este estudo clínico prospectivo foi aprovado pelo Comitê de Ética em Pesquisa da Universidade Federal de São Paulo Escola Paulista de Medicina (UNIFESP - EPM). Foram selecionados 46 pacientes estado físico ASA I e II, com idade entre 18 e 65 anos, com índice de massa corpórea entre 20 e 25 $\mathrm{kg} \cdot \mathrm{m}^{-2}$ submetidos a procedimentos cirúrgicos eletivos sob anestesia geral com intubação traqueal e ventilação mecânica controlada.

Após o consentimento informado por escrito, os pacientes foram distribuídos aleatoriamente em dois grupos: GC (controle) e GL (lidocaína). Todos os pacientes receberam rocurônio como bloqueador neuromuscular. No GL os pacientes receberam lidocaína, três minutos antes de receberem o rocurônio. No GC os pacientes receberam igual volume de solução fisiológica. Constituiu critério de exclusão a presença de situações que interferissem na ação dos bloqueadores neuromusculares tais como hipo ou hiperproteinemia, insuficiência renal, hepatopatias, hipo ou hipertermia, alterações do equilíbrio ácido-base e hidroeletrolítico, gravidez, doenças neuromusculares, alcoolismo crônico e fármacos que alteram a farmacologia dos BNM.

Os pacientes não receberam medicação pré-anestésica. Um acesso venoso foi obtido no membro superior com cateter $18 \mathrm{G}$ para hidratação e administração de fármacos. Foi instalada uma torneira diretamente no cateter de forma a possibilitar que os fármacos atingissem a corrente sangüínea para excluir interferência do calibre e comprimento da extensão no tempo de infusão da lidocaína e do rocurônio. Utilizou-se como monitorização o cardioscópio, oxímetro de pulso, capnógrafo e analisador de gases, monitor não-invasivo de pressão arterial, monitor da transmissão neuromuscular pelo método da acelerografia (TOF GUARD ${ }^{\circledR}$ ), termômetros esofágico e cutâneo.

A indução da anestesia foi realizada com fentanil $\left(5 \mu \mathrm{g} \cdot \mathrm{kg}^{-1}\right)$, seguida de etomidato $\left(0,3 \mathrm{mg} \cdot \mathrm{kg}^{-1}\right)$, após três minutos. Mantida ventilação manual sob máscara com oxigênio a $100 \%$ e isoflurano com fração expirada de, no máximo, 0,5\% até a estabilização da resposta do músculo adutor do polegar à estimulação elétrica do nervo ulnar. Prosseguiu-se a indução com a administração de $1,5 \mathrm{mg} \cdot \mathrm{kg}^{-1}$ de lidocaína (GL) ou volume equivalente de solução fisiológica (GC). Após três minutos administrou-se $0,6 \mathrm{mg} \cdot \mathrm{kg}^{-1}$ de rocurônio, em 15 segun- dos. A laringoscopia e a intubação traqueal foram realizadas após o desaparecimento das quatro respostas à seqüência de quatro estímulos (SQE).

A anestesia foi mantida com isoflurano em concentração de até $1 \%$ de fração expirada, em oxigênio e óxido nitroso a $50 \%$. Doses adicionais de $2 \mu \mathrm{g} . \mathrm{kg}^{-1}$ de fentanil foram administradas quando a pressão arterial ou a freqüência cardíaca atingiam valores acima de $20 \%$ dos basais. A temperatura central foi mantida entre 36,5 e $37^{\circ} \mathrm{C}$ e a periférica acima de $34^{\circ} \mathrm{C}$ durante todo o procedimento, utilizando manta térmica com calor por convecção. A ventilação mecânica foi ajustada para manter a fração expirada de $\mathrm{CO}_{2}$ ao final da expiração $\left(\mathrm{P}_{\mathrm{ET}} \mathrm{CO}_{2}\right)$ entre 32 e $36 \mathrm{mmHg}$.

A função neuromuscular (FNM) foi monitorizada de forma contínua no membro superior contralateral ao do acesso venoso, pelo monitor TOF-Guard (Dinamark), utilizando o padrão de estimulação SQE (seqüência de quatro estímulos), através da estimulação supramáxima do nervo ulnar por dois eletrodos de superfície localizados no punho. Esta estimulação foi mantida por no mínimo cinco minutos para a estabilização da resposta do músculo adutor do polegar. O grau de bloqueio neuromuscular foi quantificado em termos de percentual da amplitude da resposta padrão inicial $T_{1} / T_{0}$ onde $T_{1}$ representa a amplitude da primeira resposta à $S Q E$ e $T_{0}$, a amplitude da primeira resposta prévia à administração do BNM.

Após a administração do rocurônio, foi observado o tempo necessário para abolição de $90 \%$ da resposta muscular $\left(T_{1}=\right.$ $10 \%$ ); o tempo necessário para abolição total da resposta muscular $\left(\mathrm{T}_{1}=0\right)$ e os tempos para recuperação de $25 \%, 75 \%$ e $95 \%$ de $T_{1}$. Também foi anotado o tempo para que a relação entre $T_{4}$ e $T_{1}$ atingisse o valor de 0,8 $\left(T_{4} / T_{1}=0,8\right)$.

Os valores de pressão arterial média, freqüência cardíaca, temperaturas periférica e central foram registrados nos momentos em que foram anotados os dados referentes à monitorização da função neuromuscular.

Os conceitos para os parâmetros analisados foram:

1. Início de ação: tempo decorrido para abolição de $100 \%$ da resposta muscular, $T_{1}=0$, após o término da injeção do bloqueador neuromuscular;

2. Duração clínica (Dur $25 \%$ ): intervalo de tempo em minutos, entre o final da injeção do rocurônio e a recuperação espontânea de $25 \%$ da primeira resposta à SQE;

3. Dur ${ }_{75 \%}$ : intervalo de tempo em minutos, decorrido entre a administração do rocurônio e a recuperação de $75 \%$ da primeira resposta à SQE

4. Duração total de ação (Dur ${ }_{95 \%}$ ): intervalo de tempo em minutos, decorrido entre a administração do rocurônio e a recuperação de $95 \%$ da primeira resposta à SQE

5. Índice de recuperação $\left(\mathrm{IR}_{25-75 \%}\right)$ : tempo decorrido entre a recuperação espontânea de $25 \%$ e $75 \%$ da altura da primeira resposta à $\mathrm{SQE}$;

6. Relação $T_{4} / T_{1}=0,8$ : intervalo de tempo em minutos, decorrido entre a administração do rocurônio e a recuperação de $80 \%$ da quarta resposta em relação à primeira resposta à SQE. 
7. Intervalo $T_{4} / T_{1}=0,8-D_{2} r_{25}$ : intervalo de tempo entre a Dur $_{25 \%}$ e a SQE atingir 0,8.

Os parâmetros - índice de massa corpórea, idade, freqüência cardíaca e pressão arterial - foram analisados através do teste $t$ de Student. A distribuição entre os sexos foi comparada através do teste de proporção de Fisher. Para a análise estatística dos tempos referentes à monitorização da função neuromuscular empregou-se o teste não paramétrico de Mann-Whitney. Para todas as variáveis, o nível de significância adotado foi de $5 \%(p \leq 0,05)$.

\section{RESULTADOS}

Foram incluídos 23 pacientes em cada grupo. As médias e desvios-padrão dos parâmetros demográficos (idade, índice de massa corpórea e sexo) encontram-se na tabela I. Com relação a estes parâmetros, os grupos mostraram-se homogêneos do ponto de vista estatístico.

Tabela I - Dados Demográficos

\begin{tabular}{lcc}
\hline & GC & GL \\
\hline Número de pacientes & 23 & 23 \\
Sexo & & \\
$\quad$ Masculino & 10 & 9 \\
$\quad$ Feminino & 13 & 14 \\
Idade * & $32,8 \pm 9,7$ & $34,1 \pm 8,8$ \\
Índice de massa corpórea * & $22,4 \pm 1,9$ & $23,1 \pm 1,7$ \\
\hline
\end{tabular}

* Valores em Média \pm DP

Não houve diferença estatística significativa entre os grupos

Na figura 1 está a média e o desvio-padrão das variáveis referentes à instalação do bloqueio neuromuscular. O tempo para abolição de $90 \%$ da resposta muscular $\left(T_{1}=10 \%\right)$, em

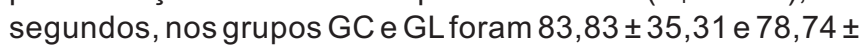
20,37 , respectivamente; sem diferença estatística significativa entre os grupos $(p=0,8175)$. Os valores do início de ação $\left(T_{1}=0\right)$, em segundos, nos grupos GC e GL foram 123,17 \pm 55,57 e $107,79 \pm 34,70$, respectivamente. Não houve diferença estatística significativa entre os grupos $(p=0,5530)$.

A figura 2 apresenta os dados referentes à fase de recuperação do bloqueio neuromuscular.

Os valores da duração clínica (Dur $25 \%$ ), em minutos, nos grupos GC e GL foram 32,35 $\pm 6,26$ e 36,48 $\pm 6,69$, respectivamente. Os grupos mostraram-se diferentes de forma estatística significativa ( $p=0,0449)$.

Os valores da duração $75 \%$ (Dur ${ }_{75 \%}$ ), em minutos, nos grupos GC e GL foram 44,09 $\pm 11,16$ e 53,83 $\pm 12,49$, respectivamente. Houve diferença estatística significativa entre os grupos $(p=0,0029)$.

Os valores da duração de ação (Dur ${ }_{95 \%}$ ), em minutos, nos grupos GC e GL foram 49,66 $\pm 13,94$ e 60,69 $\pm 13,82$. Os grupos mostraram-se estatisticamente diferentes $(p=0,0060)$.

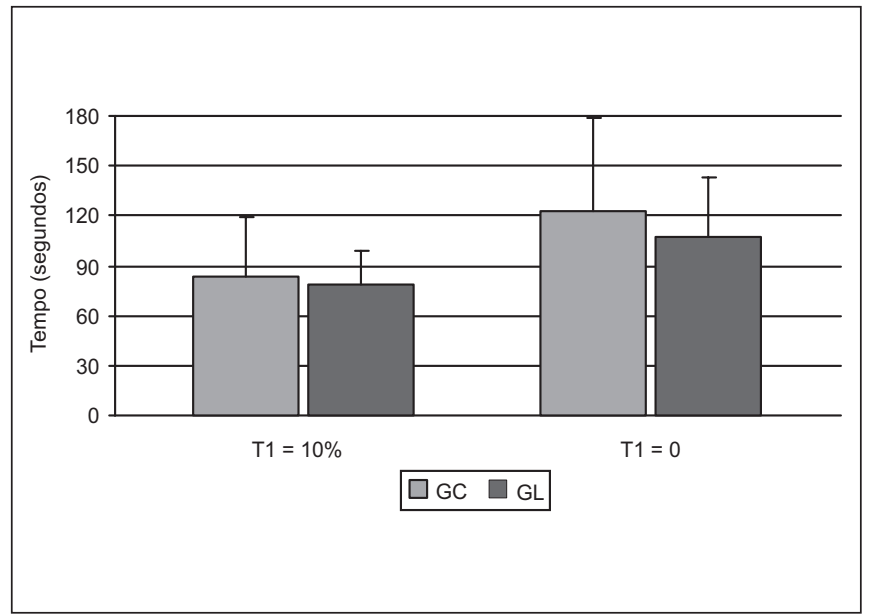

Figura 1 - Tempo (Segundos), para a Instalação do Bloqueio Neuromuscular nos Grupos Controle (GC) e Lidocaína (GL) Não houve diferença entre os grupos

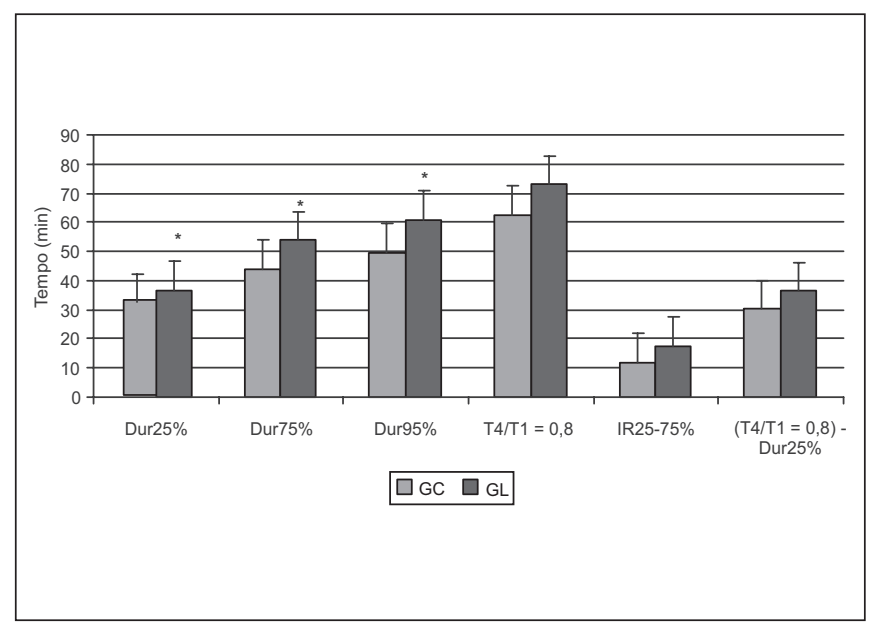

Figura 2 - Tempo( Minutos), para a Recuperação do Bloqueio Neuromuscular nos Grupos Controle (GC) e Lidocaína (GL) ${ }^{*} p=0,05$ em relação ao Grupo Controle

As médias e os desvios-padrão do índice de recuperação ( $\left(R_{25-75}\right)$, em minutos, foram $11,74 \pm 6,50$ em GC e 17,35 \pm 9,90 em GL. Não houve diferença estatística significativa entre os grupos $(p=0,2531)$.

As médias e os desvios-padrão do tempo para a relação $\mathrm{T}_{4} / \mathrm{T}_{1}$ atingir 0,8 , em minutos, foram $62,52 \pm 15,31$ em GC e $72,87 \pm 17,21$ em GL. Não houve diferença estatística significativa entre os grupos ( $p=0,0558)$.

As médias e os desvios-padrão dos intervalos de tempo entre a relação $T_{4} / T_{1}$ atingir 0,8 e a Dur $25 \%$, em minutos, foram $30,17 \pm 11,21$ em GC e 36,39 $\pm 14,17$ em GL. Não houve diferença estatística significativa entre os grupos $(p=0,1378)$.

A figura 3 apresenta os dados referentes à pressão arterial média e à freqüência cardíaca nos diferentes momentos em que foram anotados os parâmetros da monitorização da fun- 


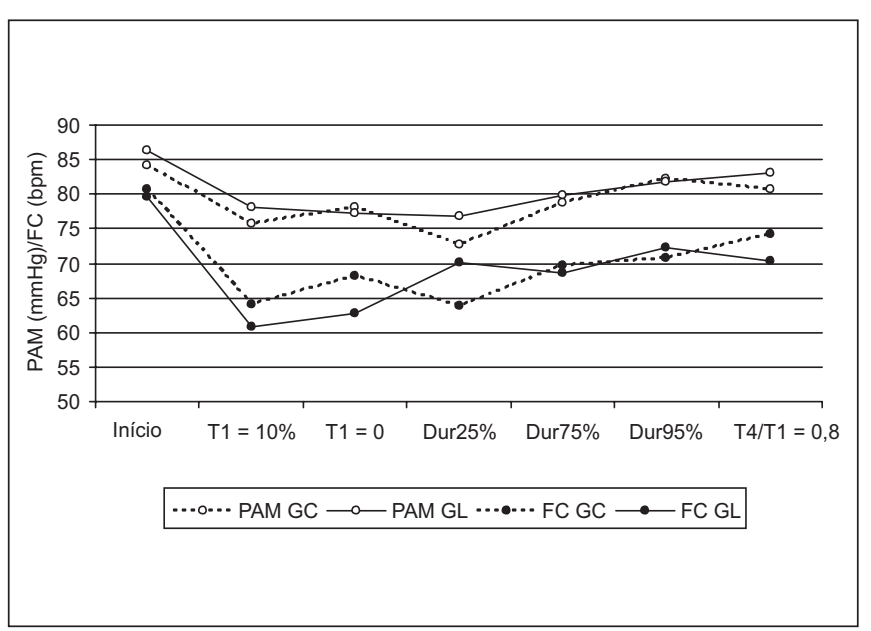

Figura 3 - Comportamento Hemodinâmico, onde PAM = Pressão Arterial Média, em mmHg e FC = Freqüência Cardíaca, em bpm

Não houve diferença estatística significativa entre os grupos

ção neuromuscular. Não houve diferenças estatística significativas entre os grupos.

\section{DISCUSSÃo}

O presente estudo foi desenhado seguindo-se as orientações do GCRP (Good Clinical Research Practice in pharmacodynamic studies of neuromuscular blocking agents) ${ }^{11}$.

Para a realização do estudo procurou-se homogeneizar os fatores que pudessem interferir com o bloqueio neuromuscular durante a anestesia.

Não se utilizou medicação pré-anestésica com benzodiazepínicos, por serem agentes capazes de causar relaxamento muscular, potencializando os $\mathrm{BNM}^{12}$. Para o intra-operatório procurou-se utilizar fármacos que priorizassem a estabilidade hemodinâmica e assegurassem a manutenção de adequada perfusão muscular, para que não houvesse interferência na distribuição do bloqueador neuromuscular até a sua biofase ${ }^{13}$. Com esta finalidade optou-se por etomidato como hipnótico para a indução anestésica.

Os anestésicos inalatórios também potencializam, por diversos mecanismos, os bloqueadores neuromusculares. Eles deprimem o sistema nervoso central, aumentam o fluxo sangüíneo muscular, diminuem a filtração glomerular, diminuem o fluxo sangüíneo hepático, diminuem a sensibilidade da membrana pós-juncional à despolarização e têm ação direta sobre a fibra muscular ${ }^{14-16}$. Dentre os agentes inalatórios, o óxido nitroso apresenta menor interação com o bloqueio neuromuscular. Autilização de isoflurano em concentrações menores que $1 \%$, associado ao óxido nitroso, foi estabelecida porque até esta concentração seu efeito sobre a função neuromuscular é comparável ao da anestesia venosa, considerada padrão para os estudos com BNM ${ }^{17}$.

Durante o ato anestésico-cirúrgico é comum a ocorrência de hipotermia ${ }^{18,19}$. O controle rígido das temperaturas central e periférica é particularmente importante porque a farmacolo- gia dos BNM e a monitorização da função neuromuscular sofrem interferências da hipotermia central e periférica ${ }^{11,20}$, respectivamente. A hipotermia potencializa a ação dos BNM por diminuir a condução nervosa, as excreções urinária e biliar dos fármacos e a atividade enzimática ${ }^{21,22}$. Sabe-se que durante a monitorização da função neuromuscular, a diminuição da temperatura da pele abaixo de $32^{\circ} \mathrm{C}$ diminui a amplitude das respostas evocadas enquanto, o calor local resulta em diminuição da impedância do eletrodo ${ }^{23-25}$. Os cuidados estabelecidos no protocolo para a manutenção das temperaturas central e periférica permitem descartar a possibilidade de interferência desse fator nos resultados obtidos.

Após a indução da anestesia os pacientes foram submetidos à ventilação manual por, no mínimo, 5 minutos antes de receberem o BNM, com a finalidade de obter estabilização da resposta muscular ao estímulo elétrico. Este cuidado é importante porque o grau de bloqueio neuromuscular é estimado comparando-se a amplitude da contração muscular isolada com um valor controle medido na ausência de bloqueio neuromuscular. Na prática, este valor de controle é geralmente difícil de ser determinado porque a estimulação repetitiva do nervo motor aumenta a resposta mecânica evocada do músculo correspondente, resultando num aumento da resposta ao estímulo isolado. Isto é conhecido como potencialização do estímulo isolado ou staircase phenomenon. A provável explicação para este fenômeno é a fosforilação da cadeia leve da miosina criando um aumento na força de contração isolada para uma determinada quantidade de cálcio liberada a cada potencial de ação. Aimplicação prática deste fenômeno é que o anestesiologista não familiarizado com esta potencialização pode considerar que, quando a resposta ao estímulo isolado atinge os níveis pré-bloqueio, a recuperação está completa ${ }^{26}$.

Aconcentração de $\mathrm{CO}_{2}$ ao final da expiração foi mantida dentro de uma faixa estreita, porque a alcalose respiratória antagoniza os efeitos dos BNM, enquanto a acidose respiratória os potencializa ${ }^{27}$.

Controlando-se todas estas variáveis e tornando os grupos homogêneos, foi possível estudar, como única variável, os efeitos da administração de lidocaína por via venosa sobre a farmacodinâmica do rocurônio.

Nas situações onde há necessidade de relaxamento muscular de instalação rápida, a utilização de succinilcolina tem sido cada vez mais substituída por outras técnicas, em decorrência dos efeitos adversos deste $\mathrm{BNM}^{28}$. As técnicas que têm sido empregadas com a finalidade de encurtar o início de ação dos BNM não-despolarizantes incluem o aumento da dose (acima de duas vezes a $\left.D E_{95}\right)^{29}$ e a dose preparatória (priming) ${ }^{30}$. Estas técnicas apresentam inconvenientes como o prolongamento da duração do bloqueio neuromuscular associado a possível efeito cardiovascular e risco de broncoaspiração, quando se utiliza aumento da dose ou dose preparatória, respectivamente ${ }^{31}$.

A busca por bloqueadores neuromusculares que pudessem substituir a succinilcolina nesta função resultou na introdução do rocurônio, o BNM não-despolarizante de mais rápido início de ação ${ }^{32}$. A fim de encurtar ainda mais seu início de 
ação, tem-se utilizado a técnica de aumento da dose (3 a 4 vezes a $\left.D E_{95}\right)^{31}$, o que permite intubação traqueal em aproximadamente 60 segundos, porém com um aumento considerável em seu tempo de ação, fazendo este BNM de ação intermediária comportar-se como BNM de longa duração ${ }^{1}$. No presente estudo procurou-se avaliar os efeitos da interação do anestésico local lidocaína com o rocurônio.

Diversos estudos demonstraram a interação sinérgica dos anestésicos locais com os BNM in vitro ${ }^{9}$ e in vivo por via venosa ${ }^{33,34}$ e por via peridural ${ }^{35,36}$.

Os anestésicos locais podem interferir com a função neuromuscular exercendo sua ação em diferentes componentes da junção mioneural, tanto na membrana pré quanto na pós-sináptica. Na membrana pré-sináptica os anestésicos locais são capazes de bloquear a condução das fibras terminais do nervo motor ${ }^{9,37}$, diminuir o conteúdo quantal de acetilcolina ou o número de quanta liberado em repouso ou à estimulação nervosa ${ }^{9,38}$ e prolongar o período refratário absoluto e a fadiga à estimulação tetânica ${ }^{37}$. Na membrana pós-sináptica, são capazes de se ligar a áreas específicas dos receptores nicotínicos diferentes das de ACh, promovendo a dessensibilização destes receptores ${ }^{39,40}$; causar bloqueio de canal aberto nos receptores nicotínicos, onde parecem se ligar a uma área específica localizada a três quartos do caminho transmembrana ${ }^{41,42}$; interferir diretamente na fibra muscular, seja por bloqueio de canais de sódio (procaína) ${ }^{43}$ ou canais de sódio e potássio (lidocaína) ${ }^{44}$. Assume-se que, durante a monitorização da função neuromuscular, a depressão e a recuperação da força de contração ao primeiro estímulo da SQE ( $T_{1}$ ou twitch) decorram dos efeitos na membrana pós-sináptica, enquanto o fenômeno da fadiga relaciona-se aos efeitos pré-sinápticos ${ }^{45}$.

Embora o desenho do presente estudo não permita avaliar os reais mecanismos da interação entre a lidocaína e o rocurônio, os resultados permitem especular sobre as possíveis ações em áreas pré e pós-sinápticas.

Em um estudo recente, os autores ${ }^{46}$ compararam as condições de intubação da succinilcolina com rocurônio $(0,6$ $\left.\mathrm{mg} \cdot \mathrm{kg}^{-1}\right)$ associado ou não à lidocaína $\left(1,5 \mathrm{mg} \cdot \mathrm{kg}^{-1}\right)$, após 60 e 90 segundos da administração do BNM. Demonstraram que a associação de lidocaína ao rocurônio permite condições apropriadas de intubação traqueal em 60 segundos, à semelhança da succinilcolina e de forma superior à administração isolada de rocurônio. Após 90 segundos todos os grupos apresentaram condições adequadas de intubação. Estes autores fundamentaram-se em critérios clínicos para definir as condições de intubação, sem realizar a monitorização da função neuromuscular dos pacientes. Nesse estudoa administração de lidocaína não foi capaz de acelerar o início de ação do rocurônio $\left(T_{1}=10 \%\right.$ e $\left.T_{1}=0\right)$, o que não necessariamente contradiz o estudo anterior, uma vez que condições adequadas de intubação podem ser obtidas mesmo na ausência de bloqueio neuromuscular ${ }^{47}$. Os autores ressaltam que esta associação apresenta a vantagem de encurtar o início de ação do rocurônio, sem o inconveniente aumento da duração de ação. No entanto, deve-se destacar que estes autores não incluíram em seu método qualquer técnica de avaliação objetiva da duração do bloqueio.

No presente estudo, a associação de lidocaína ao rocurônio promoveu aumento nas durações 25\%, 75\% e 95\% do bloqueio. Como não foram avaliados os efeitos da associação de lidocaína com diferentes doses de rocurônio, o método não permite comparar se o efeito da associação dos dois fármacos assemelha-se ao aumento da dose. Entretanto, considerando-se os resultados obtidos, a associação promoveu o retardo da recuperação inicial do bloqueio neuromuscular, sem prolongar a recuperação final.

Em outro estudo, que empregou método semelhante ao desta pesquisa, os autores demonstraram que a administração de lidocaína $\left(1,5 \mathrm{mg} \cdot \mathrm{kg}^{-1}\right)$, três minutos antes do BNM, foi capaz de encurtar o início de ação do vecurônio. No entanto, esses autores não investigaram o impacto da co-administração destes dois fármacos sobre a duração do BNM ${ }^{48}$.

Com relação à fase de recuperação do bloqueio neuromuscular, em concordância com os dados de literatura, no presente estudo a lidocaína prolongou o tempo de recuperação da primeira resposta à SQE, representadas por Dur $_{25 \%}$, $\operatorname{Dur}_{75 \%}$ e Dur ${ }_{95 \%}$, o que ratifica seus efeitos pós-sinápticos. A lidocaína retardou o início da recuperação, deslocando os valores a partir da Dur $25 \%$ de forma uniforme, o que pode ser evidenciado pela ausência de diferença no índice de recuperação $\left(\mathrm{IR}_{25-75 \%}\right)$.

Em contrapartida, o presente estudo não detectou ação pré-sináptica significativa na interação da lidocaína com o rocurônio, evidenciada pela ausência de diferença entre os tempos para a recuperação final da função neuromuscular, representados pelos tempos para $T_{4} / T_{1}$ atingir 0,8 e a diferença entre os tempos para $T_{4} / T_{1}=0,8$ e Dur $25 \%$, que tem sido proposto como índice da velocidade da recuperação final ${ }^{11}$. Considerando que os BNM não-despolarizantes apresentam diferentes afinidades com os receptores pré e pós-sinápticos e que a lidocaína atua dificultando a transmissão neuromuscular no terminal pré-sináptico e na fibra muscular, podemos concluir, nas condições estudadas, que o anestésico local interferiu com maior intensidade na intensificação dos efeitos pós-sinápticos.

Nas condições estudadas, a administração por via venosa de lidocaína previamente ao rocurônio não foi capaz de encurtar o início de ação deste BNM, porém prolongou sua duração farmacológica sem prolongar o tempo para a recuperação completa da função neuromuscular. 


\section{Effects of Intravenous Lidocaine on the Pharmacodynamics of Rocuronium}

Leandro Sotto Maior Cardoso, TSA, M.D.; César Romão Martins, TSA, M.D.; Maria Angela Tardelli, TSA, M.D.

\section{INTRODUCTION}

Rocuronium bromide is an intermediate nondepolarizing aminosteroid neuromuscular blocker $(\mathrm{NMB})^{1-5}$. Among current nondepolarizing MNBs, rocuronium has the shortest onset ${ }^{6}$, being indicated when rapid tracheal intubation without the inconveniences of succinylcholine is desired ${ }^{7}$.

Lidocaine is a popular intravenous agent for anesthetic induction, which decreases hemodynamic responses associated to tracheal intubation ${ }^{8}$.

There are evidences that local anesthetics decrease neuromuscular transmission with effects on motor neurons and muscle fibers. In vitro studies have shown that the association of these agents to NMB results in improved NMB effects ${ }^{9,10}$. In clinical practice, this synergistic effect of NMB and local anesthetics could be useful if it resulted in early neuromuscular block installation, which would provide faster tracheal intubation and protection against cardiovascular effects inherent of the technique.

This study aimed at evaluating whether intravenous lidocaine preceding rocuronium for tracheal intubation would change the pharmacodynamics of this neuromuscular blocker.

\section{METHODS}

This clinical prospective study was approved by the $\mathrm{Re}$ search Ethics Committee, Universidade Federal de São Paulo, Escola Paulista de Medicina (UNIFESP - EPM) and involved 46 patients aged 18 to 65 years, physical status ASA I and II, with body mass index between 20 and $25 \mathrm{~kg} \cdot \mathrm{m}^{-2}$, submitted to elective surgical procedures under general anesthesia with tracheal intubation and mechanically controlled ventilation.

After their written and informed consent, patients were randomly distributed in two groups: CG (control) and LG (lidocaine). All patients were given rocuronium for neuromuscular block. LG patients received lidocaine 3 minutes before rocuronium, while GC patients received equal volume of saline solution. Exclusion criteria were situations interfering with neuromuscular blocker action, such as hypo or hyperproteinemia, renal failure, liver diseases, hypo or hyperthermia, changes in acid-base and hydroelectrolytic balance, pregnancy, neuromuscular diseases, chronic alcoholism and drugs affecting NMB pharmacology.

Patients were given no premedication. Venous access was obtained in the arm with $18 \mathrm{G}$ catheter for hydration and drug administration. Atap was directly connected to the catheter to allow drugs to directly reach blood flow and rule out the influ- ence of catheter size and length on lidocaine and rocuronium infusion time.

Monitoring consisted of cardioscopy, pulse oximetry, capnography, blood gases analyzer, noninvasive blood pressure, neuromuscular transmission monitoring by acceleromyography (TOF GUARD ${ }^{\circledR}$ ), and esophageal and cutaneous thermometers.

Anesthesia was induced with fentanyl $\left(5 \mu \mathrm{g} \cdot \mathrm{kg}^{-1}\right)$ and etomidate $\left(0.3 \mathrm{mg} \cdot \mathrm{kg}^{-1}\right)$, followed three minutes later by rocuronium. Patients were maintained under manual ventilation with $100 \%$ oxygen and isoflurane and expired fraction up to $0.5 \%$ until stabilization of adductor pollicis response to electric ulnar nerve stimulation. Induction went on with 1.5 $\mathrm{mg} \cdot \mathrm{kg}^{-1}$ lidocaine (LG) or equivalent volume of saline (CG). Rocuronium $\left(0.6 \mathrm{mg} . \mathrm{kg}^{-1}\right)$ was administered three minutes later during 15 seconds. Laryngoscopy and tracheal intubation was performed after abolishment of TOF responses.

Anesthesia was maintained with isoflurane to a maximum of $1 \%$ expired fraction, mixed in oxygen and $50 \%$ nitrous oxide. Additional $2 \mu \mathrm{g} . \mathrm{kg}^{-1}$ fentanyl was administered whenever blood pressure or heart rate would go beyond $20 \%$ of baseline values. Central temperature was maintained between 36.5 and $37^{\circ} \mathrm{C}$ and peripheral temperature was maintained above $34{ }^{\circ} \mathrm{C}$ throughout the procedure with the aid of thermal blanket with convection heat. Mechanical ventilation was adjusted to maintain end tidal $\mathrm{CO}_{2}\left(\mathrm{P}_{\mathrm{ET}} \mathrm{CO}_{2}\right)$ between 32 and 36 $\mathrm{mmHg}$.

Neuromuscular function (NMF) was continuously monitored in the arm contralateral to intravenous access with TOF Guard (Denmark) monitor, using TOF stimulation (train of four), through supramaximal stimulation of the ulnar nerve with two surface electrodes on the wrist. This stimulation was maintained for at least 5 minutes to stabilize adductor pollicis muscle response. Neuromuscular block was quantified as the percentage of standard initial $T_{1} / T_{0}$ response, where $T_{1}$ is the amplitude of first TOF response and $T_{0}$ is the amplitude of the first response before NMB administration.

After rocuronium administration, time needed to abolish $90 \%$ of muscle response $\left(T_{1}=10 \%\right)$, time needed for total muscle response abolishment $\left(T_{1}=0\right)$ and $25 \%, 75 \%$ and $95 \%$ recovery time for $T_{1}$ were registered. Time for $T_{4} / T_{1}$ ratio to reach $0.8\left(T_{4} / T_{1}=0,8\right)$ was also recorded.

Mean blood pressure, heart rate, peripheral and central temperature were recorded simultaneously to data on neuromuscular function monitoring.

Definitions of the evaluated parameters were:

1. Onset: time for $100 \%$ muscle response abolishment, $T_{1}=$ 0 , after end of neuromuscular blocker injection;

2. Clinical duration (Dur $25 \%$ ): time in minutes between end of rocuronium injection and spontaneous recovery of $25 \%$ of first TOF response;

3. Dur ${ }_{75 \%}$ : time in minutes between rocuronium injection and $75 \%$ recovery of first TOF response; 
4. Total duration of action ( Dur $_{95 \%}$ ): time in minutes between rocuronium injection and $95 \%$ recovery of first TOF response;

5. Recovery index $\left(\mathrm{RI}_{25-75 \%}\right)$ : time between $25 \%$ and $75 \%$ spontaneous recovery of first TOF response height;

6. Ratio $T_{4} / T_{1}=0.8$ : time in minutes between rocuronium injection and $80 \%$ recovery of fourth TOF response as compared to first response;

7. Interval $\mathrm{T}_{4} / \mathrm{T}_{1}=0.8$ : - Dur $25 \%$ : interval between $\operatorname{Dur}_{25 \%}$ and 0.8 TOF.

Student's $t$ test was used to evaluate body mass index, age, heart rate and blood pressure. Fisher's proportion test was used to compare gender distribution. Mann-Whitney's non-parametric test was used for statistical analysis of neuromuscular function monitoring. For all variables, significance level was $5 \%(p \leq 0.05)$.

\section{RESULTS}

There were 23 patients in each group. Demographic data means and standard deviations (age, body mass index and gender) are shown in table I, and were statistically similar.

Table I - Demographics Data

\begin{tabular}{lcc}
\hline & CG & LG \\
\hline Number of patients & 23 & 23 \\
Gender & 10 & 9 \\
$\quad$ Male & 13 & 14 \\
$\quad$ Female & $32.8 \pm 9.7$ & $34.1 \pm 8.8$ \\
Age * & $22.4 \pm 1.9$ & $23.1 \pm 1.7$ \\
Body mass index* & &
\end{tabular}

* Values expressed in Mean \pm SD

There were no statistically significant differences between groups

Figure 1 shows mean and standard deviation of neuromuscular block installation variables. Time for $90 \%$ muscle response abolishment $\left(T_{1}=10 \%\right)$, in seconds, for groups CG and LG were $83.83 \pm 35.31$ and $78.74 \pm 20.37$ respectively, without statistically significant differences $(p=$ $0.8175)$. Onset $\left(T_{1}=0\right)$, in seconds, for groups CG and LG was $123.17 \pm 55.57$ and $107.79 \pm 34.70$ respectively, without statistically significant differences $(p=0.5530)$.

Figure 2 shows neuromuscular block recovery data.

Clinical duration (Dur $25 \%$ ), in minutes for groups CG and LG was $32.35 \pm 6.26$ and $36.48 \pm 6.69$ respectively, with statistically significant difference $(p=0.0449)$.

Values of $75 \%$ duration (Dur $75 \%$ ), in minutes, for groups GC and LG were $44.09 \pm 11.16$ and $53.83 \pm 12.49$, respectively, with statistically significant difference $(p=0.0029)$.

Duration of action (Dur ${ }_{95 \%}$ ), in minutes, for groups GC and LG was $49.66 \pm 13.94$ and $60.69 \pm 13.82$, with statistically significant difference $(p=0.0060)$.

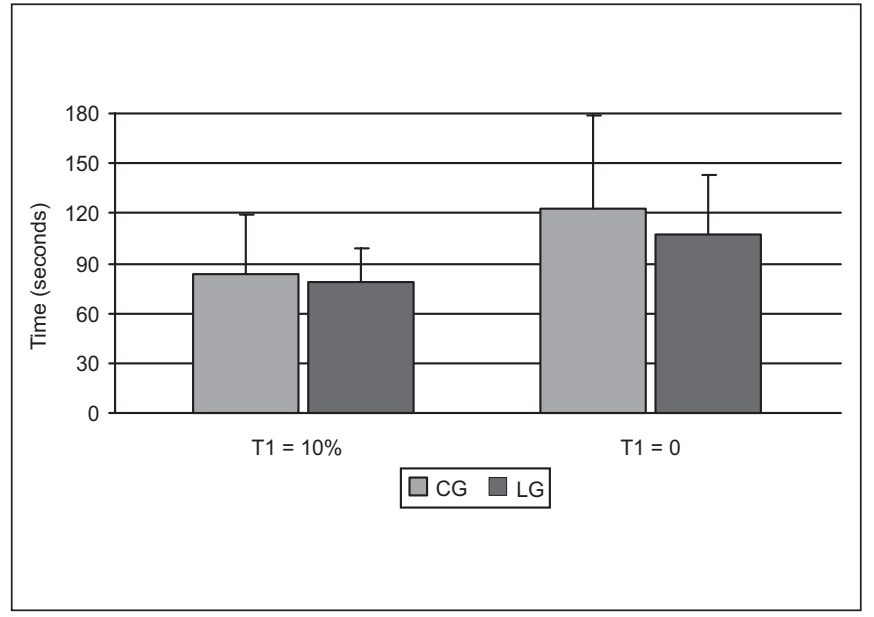

Figure 1 - Time ( Seconds), for Neuromuscular Block Installation in Control (CG) and Lidocaine (LG) Groups There were no differences between groups

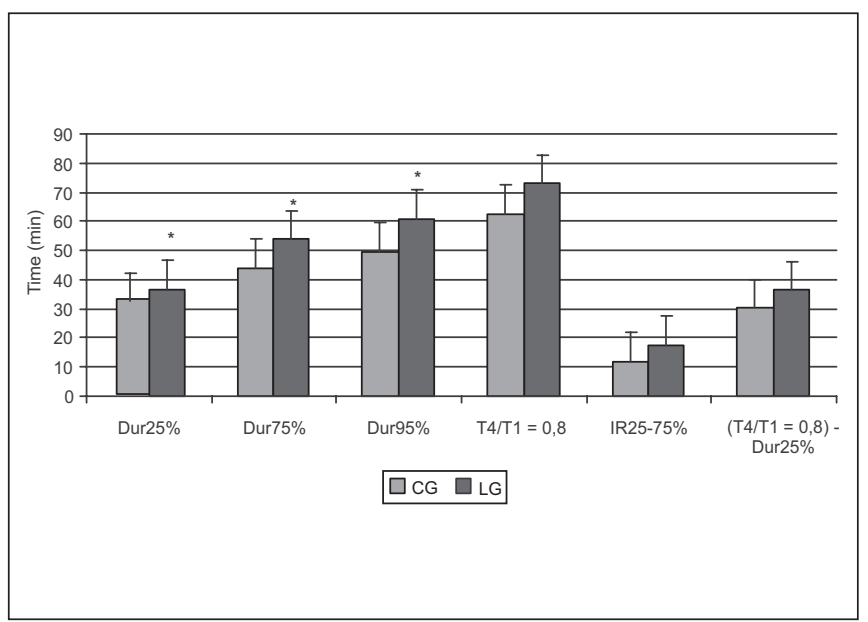

Figure 2 - Time (Minutes) for Neuromuscular Block Recovery in Control (CG) and Lidocaine (LG) Groups ${ }^{*} p=0.05$ as compared to Control Group

Recovery index $\left(\mathrm{RI}_{25-75}\right)$ means and standard deviations, in minutes, were $11.74 \pm 6.50$ for $C G$ and $17.35 \pm 9.90$ for $L G$, without statistically significant differences $(p=0.2531)$.

Means and standard deviations of time for $\mathrm{T}_{4} / \mathrm{T}_{1}$ ratio to reach 0.8 , in minutes, were $62.52 \pm 15.31$ for $C G$ and $72.87 \pm 17.21$ for LG, without statistically significant differences $(p=$ 0.0558).

Means and standard deviations of time intervals between $\mathrm{T}_{4} / \mathrm{T}_{1}$ ratio to reach 0.8 and $\mathrm{Dur}_{25 \%}$, in minutes, were $30.17 \pm$ 11.21 for $C G$ and $36.39 \pm 14.17$ for LG, without statistically significant differences $(p=0.1378)$.

Figure 3 shows mean blood pressure and heart rate in the moments in which neuromuscular function monitoring parameters were recorded. There were no statistically significant differences between groups. 


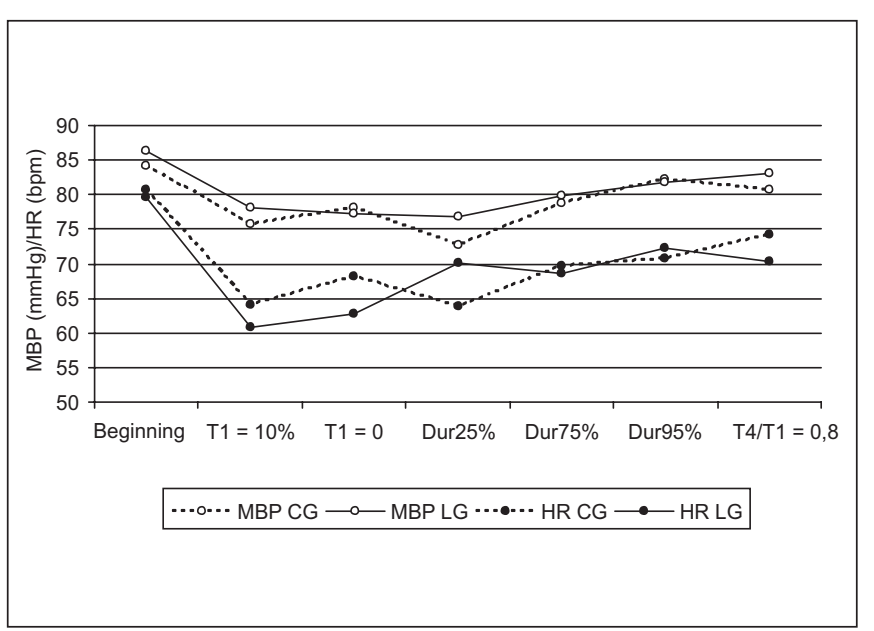

Figure 3 - Hemodynamic Behavior, where MBP = Mean Blood Pressure, in $\mathrm{mmHg}$, and $\mathrm{HR}=$ Heart Rate, in bpm There were no statistically significant differences between groups

\section{DISCUSSION}

This study has followed the guidelines of GCRP (Good Clinical Research Practice in pharmacodynamic studies of neuromuscular blocking agents) ${ }^{11}$.

We have tried to standardize factors, which could interfere with neuromuscular block during anesthesia.

No benzodiazepine was used as premedication because they may promote muscle relaxation, thus potentiating NMBs

12. Intraoperatively, we have tried to use drugs prioritizing hemodynamic stability and assuring adequate muscle perfusion, so that there would be no interference in neuromuscular blocker distribution to its biophase ${ }^{13}$. This way, etomidate was chosen as hypnotic for the anesthetic induction.

Inhalational anesthetics also potentiate neuromuscular blockers by different mechanisms. They depress central nervous system, increase muscle blood flow, decrease glomerular filtration, decrease liver blood flow, and decrease post-junctional membrane sensitivity to depolarization and have direct action on muscle fibers ${ }^{14-16}$. Among inhalational anesthetic agents, nitrous oxide presents the lowest interaction with neuromuscular block. Isoflurane below $1 \%$ associated to nitrous oxide was used because, up to this concentration, its effect on neuromuscular function is similar to that of intravenous anesthesia, which is the standard for NMB trials ${ }^{17}$.

Hypothermia is common during surgical procedures ${ }^{18,19}$. Strict control of central and peripheral temperature is particularly important since NMBs pharmacology and neuromuscular function monitoring are affected by central and peripheral hypothermia, respectively ${ }^{11,20}$. Hypothermia potentiates NMB action by decreasing nervous conduction, urinary and billiary excretion and enzymes activity ${ }^{21,22}$. It is known that during neuromuscular function monitoring, decreased skin temperature to below $32^{\circ} \mathrm{C}$ reduces evoked responses amplitude, while local heat decreases electrode impedance ${ }^{23-25}$. Care taken by the protocol to maintain central and peripheral temperature allows us to rule out the interference of this factor on results.

After anesthetic induction, patients were submitted to manual ventilation for at least 5 minutes before receiving NMB, to stabilize muscle response to electric stimulation. This is important because the level of neuromuscular block is estimated by comparing the amplitude of muscle contraction and a control value measured in the absence of neuromuscular block.

Practically, this control value is in general difficult to determine because repetitive motor nerve stimulation increases mechanical evoked response of the corresponding muscle, resulting in increased response to isolated stimulation. This is known as staircase phenomenon. Possible explanation for such finding is phosphorilation of the light myosin chain increasing isolated contraction strength to a certain amount of calcium released at each action potential. Practical implication of this event is that an anesthesiologists unfamiliar with this potentiation may consider that, when isolated stimulation response reaches pre-blockade levels, recovery is complete ${ }^{26}$.

End tidal $\mathrm{CO}_{2}$ concentration was maintained within a narrow range because respiratory alkalosis antagonizes NMB effects, while respiratory acidosis precipitate them ${ }^{27}$.

By controlling these variables and making groups homogeneous, it was possible to evaluate, as a single variable, the effects of intravenous lidocaine on the pharmacodynamics of rocuronium.

When rapid muscle relaxation is desired, succinylcholine is being increasingly replaced by other techniques due to its adverse effects ${ }^{28}$. Techniques to shorten nondepolarizing NMB onset includes higher dose (more than twice $\left.D E_{95}\right)^{29}$ and priming dose ${ }^{30}$. These techniques, however, may involve undesirable effects, like prolonged neuromuscular block length, which is associated to possible cardiovascular effects or risk of bronchial aspiration, when increased dose or priming dose are used, respectively ${ }^{31}$.

The search for neuromuscular blockers to replace succinylcholine has resulted in the development of rocuronium, nondepolarizing NMB with the fastest onset ${ }^{32}$. To further shorten onset, the higher dose technique has been used ( 3 to 4 times $\left.D E_{95}\right)^{31}$, allowing tracheal intubation in approximately 60 seconds, however with considerable increase in duration, making this intermediate NMB behave as long duration $\mathrm{NMB}^{1}$.

Our study tried to evaluate the effects of the interaction of lidocaine and rocuronium.

Different studies have shown synergistic interaction of local anesthetics and NMBs in vitro and in vivo, both intravenously 33,34 and epidurally ${ }^{35,36}$.

Local anesthetics may interfere with neuromuscular function by acting on different myoneural junction components, both in pre and post-synaptic membrane. In the pre-synaptic membrane, local anesthetics block motor nerve terminal fibers conduction ${ }^{9,37}$, decrease the quantal content of acetylcholine or the number of quanta released at rest or after nervous stimulation ${ }^{9,38}$, and prolong the absolute refractory pe- 
riod and the fatigue to tetanic stimulation ${ }^{37}$. In the post-synaptic membrane, they bind to specific areas of nicotinic receptors different from ACh, promoting loss of sensitization of such receptors ${ }^{39,40}$, block open nicotinic receptors channels, where they seem to bind to a specific area located at $3 / 4$ of the transmembrane pathway ${ }^{41,42}$, and directly interfere with muscle fibers by blocking sodium channels (procaine) ${ }^{43}$ or sodium and potassium channels (lidocaine) ${ }^{44}$.

It is assumed that during neuromuscular function monitoring, depression and recovery of contraction strength to first TOF stimulation ( $T_{1}$ or twitch) is a consequence of effects on post-synaptic membrane, while fatigue is related to pre-synaptic effects $^{45}$.

Although our study design does not allow for the evaluation of actual mechanisms of lidocaine/rocuronium interaction, results do allow some speculations on possible actions on pre and post-synaptic areas.

A recent study ${ }^{46}$ has compared intubation conditions provided by succinylcholine with those after rocuronium (0.6 $\left.\mathrm{mg} \cdot \mathrm{kg}^{-1}\right)$ associated or not to lidocaine $\left(1.5 \mathrm{mg} \cdot \mathrm{kg}^{-1}\right), 60$ and 90 seconds after NMB administration. They have shown that the association lidocaine/rocuronium provides adequate tracheal intubation conditions in 60 seconds, similarly to succinylcholine and better than rocuronium alone. All groups presented adequate intubation conditions 90 seconds later. These authors were based on clinical criteria to define intubation conditions, without monitoring neuromuscular function. In our study, lidocaine was unable to shorten rocuronium onset $\left(T_{1}=10 \%\right.$ and $\left.T_{1}=0\right)$, what does not necessarily contradicts the previous study since adequate intubation conditions may be obtained even in the absence of neuromuscular blockers ${ }^{47}$. Authors stress that this association has the advantage of shortening rocuronium onset without increasing its duration. However, it should be highlighted that these authors have not included in their method any objective evaluation of blockade duration.

In our study, the association lidocaine/rocuronium has promoted increased $25 \%, 75 \%$ and $95 \%$ blockade duration. Since the effects of the association of lidocaine to different rocuronium doses were not evaluated, the method does not allow us to verify whether the effect of both drugs association is similar to the one of an increased NMB dose. However, considering our results, the association has promoted delay in early neuromuscular block recovery, without prolonging its late recovery.

In a different study with a method similar to ours, authors have shown that lidocaine $\left(1.5 \mathrm{mg}^{\mathrm{kg}}{ }^{-1}\right) 3$ minutes before NMB was able to shorten vecuronium onset. However, these authors have not investigated the impact of the co-administration of both drugs on NMB duration ${ }^{48}$.

As to neuromuscular block recovery phase, and in line with data in the literature, our study showed that lidocaine prolonged recovery time for first TOF response, represented by Dur $_{25 \%}$, Dur ${ }_{75 \%}$ and Dur ${ }_{95 \%}$, which confirms its post-synaptic effects. Lidocaine prolonged recovery by uniformly shifting values as from Dur $25 \%$, what can be seen by the lack of difference in recovery index $\left(\mathrm{RI}_{25-75 \%}\right)$.
On the other hand, our study has not detected significant pre-synaptic action of the interaction of lidocaine and rocuronium, evidenced by the lack of difference between times for final neuromuscular function recovery, represented by times for $T_{4} / T_{1}$ to reach 0.8 and the difference between times for $T_{4} / T_{1}=0.8$ and $D r_{25}$, which has been proposed as final recovery speed index ${ }^{11}$.

Considering that nondepolarizing NMBs have different affinities for pre and post synaptic receptors, and that lidocaine acts by impairing neuromuscular transmission in the pre-synaptic terminal and muscle fibers, one may conclude, in the conditions of our study, that the local anesthetic agent has more intensively interfered with post-synaptic effects.

In our conditions, intravenous lidocaine administered before rocuronium was unable to shorten its onset, but prolonged its pharmacological duration without prolonging total neuromuscular function recovery.

\section{REFERÊNCIAS - REFERENCES}

01. Booij LHD, Knape HTA - The effect of neuromuscular blockade of ORG 9426. A new muscle relaxant non-depolarizing steroid in man. Anesthesia, 1991;46:341-343.

02. Boot MG, Marsh B, Bryden FM et al - A comparison of pharmacodynamics of rocuronium and vecuronium during halothane anaesthesia. Anaesthesia, 1992;47:832-834.

03. Lambalk LM, De Wit AP, Wierda JM et al - Dose-response relationship and time course of action of Org 9426. A new muscle relaxant of intermediate duration evaluated under various anaesthetic techniques. Anaesthesia, 1991;46:907-911

04. Silverman DG - Neuromuscular Block in Perioperative and Intensive Care. Philadelphia: J.B. Lippincott, 1994;204-210.

05. Wierda JM, Proost JH, Muir AW et al - Design of drugs for rapid onset. Anaesthetic Pharmacol Review, 1993;1:57-68.

06. Wierda JM, De Wit AP, Kuizenga K et al - Clinical observations on the neuromuscular blocking action of Org 9426, a new steroidal non-depolarizing agent. Br J Anaesth, 1990;64: 521-523.

07. Magorian T, Flannery KB, Miller RD - Comparison of rocuronium, succinylcholine, and vecuronium for rapid-sequence induction of anesthesia in adult patients. Anesthesiology, 1993;79:913-918.

08 . Hamaya Y, Dohi S - Differences in cardiovascular response to airway stimulation at different sites and blockade of the response by lidocaine. Anesthesiology, 2000;93:95-103.

09. Matsuo S, Rao DB, Chaudry I et al - Interaction of muscle relaxants and local anesthetics at the neuromuscular junction. Anesth Analg, 1978;57:580-587.

10. Pederneiras SG - Interação de drogas com relaxantes musculares. Rev Bras Anestesiol, 1988;38:63-73.

11. Viby-Mogensen J, Engbaek J, Eriksson LI et al - Good clinical research practice (GCRP) in pharmacodynamic studies of neuromuscular blocking agents. Acta Anaesthesiol Scand, 1996;40:59-74.

12. Feldman SA, Crawley BE - Interaction of diazepam with muscle relaxant drugs. Br Med J, 1970;1:336-338.

13. Gill RS, Scott RP - Etomidate shortens the onset time of neuromuscular block. Br J Anaesth, 1992;69:444-446.

14. Pollar BJ, Miller RA - Potentiation and depressant effects of inhalation anaesthetics on the rat phrenic nerve-diaphragm preparation. Br J Anaesth, 1973;45:404-440.

15. Karis JH, Gissen AJ, Nastuk WL - The effect of volatile anesthetic agents on the neuromuscular transmission. Anesthesiology, 1967;28:128-134.

16. Waud BE, Waud DR - Effects of volatile anesthetics on directly and indirectly stimulated skeleton muscle. Anesthesiology, 1979;50: 103-110. 
17. Kansanaho M, Olkkola KT, Wierda JM - Dose-response and concentration-response relation of rocuronium infusion during propofol-nitrous oxide and isoflurane-nitrous oxide anesthesia. Eur J Anaesthesiol, 1997;14:488-494.

18. England AJ, Wu X, Richards KM et al - The influence of cold on the recovery of three neuromuscular blocking agents in man. Anaesthesia, 1996;51:236-240

19. Heier T, Caldwell JE, Eriksson LI et al - The effect of hypothermia on adductor pollicis twitch tension during continuous infusion of vecuronium in isoflurane- anesthetized humans. Anesth Analg, 1994;78:312-317.

20. Tardelli MA - Função Neuromuscular: Bloqueio, Antagonismo e Monitorização, em: Yamashita AM et al - Anestesiologia - SAESP. São Paulo Editora Atheneu, 2001;217-244.

21. Ham J, Stanky DR, Neufield P - Pharmacokinetics and dynamics of D tubocurarineduring hypothermia in humans. Anesthesiology, 1981;55:631-635.

22. Heier T, Caldwell JE, Sessler DI et al - The effect of local surface and central cooling on adductor policis twitch tension during nitrous oxide/isoflurane and nitrous oxide/fentanyl anesthesia in humans. Anesthesiology, 1990;72:807-811.

23. Zipp P - Temperature dependent alteration of the surface-EMG and ECG: an investigation of the electrical transfer characteristics of the human skin. Eur J Appl Physiol Occup Physiol, 1977;37:275-288.

24. Ericksson LI, Lennmarken C, Jensen E et al - Twitch tension and train-of-of-four ratio during prolonged neuromuscular monitoring a different peripheral temperatures. Acta Anaesthesiol Scand, 1991;35:247-252.

25. Smith DC, Booth JV - Influence of muscle temperature and forearm position on evoked electromyography in the hand. $\mathrm{Br} \mathrm{J}$ Anaesth, 1994;72:407-410.

26. Eleveld DJ, Kopman AF, Proost JH et al - Model to describe the degree of twich potentiation during neuromuscular monitoring. $\mathrm{Br} \mathrm{J}$ Anaesth, 2004;92:373-380.

27. Gencarelli PJ, Swen J, Koot HW et al - The effects of hypercarbia and hypocarbia on pancuronium and vecuronium neuromuscular blockades in anesthetized humans. Anesthesiology, 1983;59: 376-380.

28. Donati F, Bevan DR - Suxamethonium - current status. Clin Anaesth, 1985;3:371-385.

29. Magorian T, Flannery KB, Miller RD - Comparison of rocuronium, succinylcholine, and vecuronium for rapid-sequence induction of anesthesia of adult patients. Anesthesiology, 1993;79:913-918.

30. Schwarz S, Ilias W, Lackner F et al - Rapid tracheal intubation with vecuronium: the priming principle. Anesthesiology, 1985;62: 388-391.

31. Solera Marín J, Amoros Aramo J, Gonzales Miranda F - Formas de Administración, em: Alvarez Gomes JA, Gonzáles Miranda F, Bustamante Bozzo R - Relajantes Musculares em Anestesia y Terapia Intensiva. Madrid. Espanha. Editora Aran, 2000;9:139-146.

32. Mirakhur RK, Cooper AR, Clarke RSJ - Onset and intubation conditions of rocuronium bromide compared to those of suxamethonium Eur Anaesth, 1994;11:41-43.

33. Katz RL, Gissen AJ - Effects of intravenous and intra-arterial procaine and lidocaine on neuromuscular transmission in man. Acta Anaesthesiol Scand, 1969:36:106-113.

34. Telivuo L, Katz RL - The effects of modern intravenous local analgesics on respiration during partial neuromuscular block in man. Anaesthesia, 1970;25:30-35.

35. Toft P, Kirkegaard Nielsen $H$, Severinsen I - Effect of epidurally administered bupivacaine on atracurium-induced neuromuscular blockade. Acta Anaesthesiol Scand, 1990;34:649-652.

36. Taivainen T, Meretoja OA, Rosenberg PH - The effect of epidural bupivacaine on vecuronium-induced neuromuscular blockade in children. Acta Anaesthesiol Scand, 1994;38:453-456.

37. Usubiaga JE, Standaert F - The effect of local anesthetics on motor nerve terminals. J Pharmacol Exp Ther, 1968;159:353-361.

38. Straughan $\mathrm{F}-\mathrm{The}$ action of procaine at the neuromuscular junction. J Pharm Pharmacol, 1961;13:49-52.
39. Cohen JB, Boyd ND, Shera NS - Interactions of Anesthetics with Nicotinic Postsynaptic Membranes Isolated from Torpedo Electric Tissue, em: Fink BR - Molecular Mechanisms of Anesthesia, Progress in Anesthesiology. New York: Reaven Press, 1980;165-174.

40. Sine SM, Taylor P - Local anesthetics and histrionicotoxin are allosteric inhibitors of the acetylcholine receptor. Studies of clonal muscle cells. J Biol Chem, 1982;257:8106-8114.

41. Neher E, Steinbach JH - Local anesthetics transiently block currents through single acetylcholine-receptor channels. J Physiol, 1978;277:153-176.

42. Ruff RL - The kinetics of local anesthetic blockade of end-plate channels. Biophys J, 1982;37:625-631.

43. Straub R - Effect of local anesthetics on ion-determined resting potential changes of myelinated nerve fibers in frogs. Arch Int Pharmacodyn Ther, 1956;107:414-430.

44. Maeno T, Edwards C, Hashimura S - Difference in effects and end-plate potentials between procaine and lidocaine as revealed by voltage-clamp experiments. J Neurophysiol, 1971;34:32-46.

45. McCoy EP, Connolly FM, Mirakhur RK et al - Nondepolarizing neuromuscular blocking drugs and train-of-four fade. Can J Anesth, 1995;42:213-216.

46. Yorukoglu D, Asik Y, Okten F - Rocuronium combined with i.v. lidocaine for rapid tracheal intubation. Acta Anaesthesiol Scand, 2003:47:583-587.

47. Puhringer FK, Khuenl-Brady KS, Koller J et al - Evaluation of the endotracheal intubating conditions of rocuronium (ORG 9426) and succinylcoline in outpatient surgery. Anesth Analg, 1992;75:37-40.

48. Nonaka A, Sugawara T, Suzuki S et al - Pretreatment with lidocaine accelerates onset of vecuronium-induced neuromuscular blockade. Masui, 2002;51:880-883.

\section{RESUMEN}

Cardoso LSM, Martins CR, Tardelli MA - Efectos de la Lidocaína por Vía Venosa sobre la Farmacodinámica del Rocuronio

JUSTIFICATIVA Y OBJETIVOS: EI rocuronio es un bloqueador neuromuscular (BNM) no despolarizante de acción intermediaria que presenta un inicio de acción más rápido, indicado para situaciones donde hay necesidad de rápida intubación traqueal. La lidocaína es frecuentemente utilizada por vía venosa para disminuir las respuestas hemodinámicas asociadas a la intubación traqueal. La asociación de un BNM a un anestésico local resulta en potencialización de los efectos bloqueadores neuromusculares. El objetivo de este estudio es evaluar la interacción entre la lidocaína y a farmacodinámica del rocuronio evaluada por aceleromiografia.

MÉTODO: Pacientes estado físico ASA I y II, con edad entre 18 y 65 años, eventualmente distribuidos en dos grupos (GC: control y GL: lidocaína), recibieron rocuronio como bloqueador neuromuscular. El GL recibió lidocaína (1,5 mg. $\left.\mathrm{kg}^{-1}\right) 3$ minutos antes del rocuronio. La función neuromuscular fue evaluada por la respuesta del músculo aductor del pulgar a la secuencia de cuatro estímulos (SQE). Después de la inyección del BNM se anotaron los tiempos para la primera respuesta $\left(T_{1}\right)$ a la $S Q E$ alcanzar 10 y 0\% del valor control, y recobrar 25\%, 75\% y 95\% de la altura de la contracción (Dur $25 \%$, Dur $\left._{75 \%}, D_{1} r_{95 \%}\right)$. Se anotó también el tiempo de recuperación de $\left.T_{4} / T_{1}=0,8\right)$ y los intervalos Dur ${ }_{75 \%}-$ Dur $_{25 \%}\left(I_{25-75}\right)$ y $T_{4} / T_{1}=0,8$ - Dur $25 \%$.

RESULTADOS: Este estudio no demostró diferencia estadísticamente significativa entre los grupos cuando comparados $T_{1}=10 \%, T_{1}=0, I R_{25-75}, T_{4} / T_{1}=0,8-D_{1} r_{25 \%}$. Los tiempos para Dur $25 \%$, Dur ${ }_{75 \%}$, Dur $95 \%$ en el GL fueron estadísticamente superiores a los del GC.

CONCLUSIONES: La asociación de lidocaína al rocuronio prolongó la parte inicial de recuperación del bloqueo sin interferir con el inicio de acción o con la parte de recuperación final. 